\title{
$\mathrm{FMCW} \mathrm{MIMO}$ 레이다를 이용한 거리-각도 동시 추정 기법
}

\section{Joint Range and Angle Estimation of FMCW MIMO Radar}

\author{
김 정 훈 ${ }^{1}$ 송 성 찬 ${ }^{\star 2}$. 전 주 환 $^{3}$ \\ Junghoon $\mathrm{Kim}^{1} \cdot$ Sungchan Song*2 ${ }^{2}$ Joohwan Chun $^{3}$ \\ 요 약
}

$\mathrm{FMCW}$ 배열 안테나는 저비용 고해상도라는 장점 때문에 많은 분야에 널리 사용되고 있다. $\mathrm{FMCW}$ 배열을 이용하여 표적의 거리와 각도를 추정하기 위해서, 우선 deramped 수신신호로부터 거리-각도 행렬을 구성하고, 그 다음에 거리-각도 행렬에 2D-FFT와 같은 2차원 주파수 추정기법을 적용한다. 하지만, 이러한 주파수 추정기법은 bias 오차를 발생시키게 된다. 그 이유는 거리-각도 행렬의 두 개의 주파수가 서로 독립적이지 않기 때문이다. 따라서 FMCW 배열 안테나를 이용 하여 표적의 거리-각도 동시추정을 위한 최대우도 기반 알고리즘을 제안하고, 거리와 각도 추정에 있어서 Cramer-Rao bound에 도달함을 보인다.

\section{Abstract}

Frequency-modulated continuous wave(FMCW) radars with array antennas are widely used because of their light weight and relatively high resolution. A usual approach for the joint range and angle estimation of a target using an array FMCW radar is to create a range-angle matrix with the deramped received signal, and subsequently apply two-dimensional(2D) frequency estimation methods such as 2D fast Fourier transform on the range-angle matrix. However, such frequency estimation approaches cause bias errors since the frequencies in the range-angle matrix are not independent. Therefore, we propose a new maximum likelihood-based algorithm for joint range and angle estimation of targets using array FMCW radar, and demonstrate that the proposed algorithm achieves the Cramér-Rao bounds, both for range as well as angle estimation.

Key words: FMCW Radars, Maximum Likelihood Estimation, 2D-FFT, 2D-MUSIC, CRB, Joint Range, Angle Estimation

\section{I. 서 론}

$\mathrm{FMCW}$ 레이다는 가볍고, 전력소모가 적으며, 저비용일 뿐만 아니라 거리의 고해상도를 얻을 수 있다는 장점이 있 으며, 고도계, 차량용 레이다, 합성개구레이다(SAR) 등 근 접거리 추정분야에 널리 사용되고 있다 ${ }^{[1]} . \mathrm{FMCW}$ 레이다는
수신안테나 배열 또는 다중안테나(MIMO) 배열로 사용될 수 있다. 수신안테나 배열이나 MIMO 배열에서 우선 거리각도 행렬을 구하게 되며, $\mathrm{MIMO}$ 의 경우에는 각도의 차원 은 가상배열(virtual array)의 크기만큼 늘어난다. 표적의 거 리와 각도를 추정하기 위해, 거리-각도 행렬에 2D-FFT나 2D-MUSIC ${ }^{2]}$ 과 같은 2차원 주파수 추정기법을 적용하게 된다.

「이 연구는 한화시스템 연구비의 지원으로 연구되었음.」

카이스트 전기 및 전자공학부(Department of Electrical Engineering, KAIST)

*한화시스템(Hanwha Systems)

1: 위촉연구원, 2: 수석연구원, 3: 교수

• Manuscript received November 12, 2018 ; Revised December 3, 2018 ; Accepted January 28, 2019. (ID No. 20181112-114)

- Corresponding Author: Junghoon Kim (e-mail: junghoonk@kaist.ac.kr) 
하지만, 이러한 기존의 2차원 주파수 추정 알고리즘은 각 domain의 주파수가 서로 결합(coupled)되어 있을 경우 에 문제가 된다. 그 이유는 표적과의 거리와 비례하는, 거 리 축의 주파수는 각각의 안테나마다 조금씩 다르기 때 문이다. 이 차이는 표적과의 각도에 따라 결정된다. 이전 연구에서는 안테나마다 거리차이가 발생한다는 점을 신 호 모델에 반영하지 않았으며, 이는 불가피하게 bias 오차 를 발생시킨다.

본 논문에서는 앞서 언급한 bias 오차를 해결하기 위해 거리-각도의 동시추정을 위한 최대우도법(maximum likelihood) 알고리즘을 제안한다. 또한, 제안하는 알고리즘을 통해 구한 거리와 각도의 추정치를 기존의 기법들과 더불 어 Cramer-Rao bounds(CRB)와도 비교한다. 더 나아가, 본 알 고리즘은 거리-각도뿐만 아니라, 거리-Doppler나 각도Doppler에도 적용 가능하다. 그 이유는 거리-Doppler의 경우, pulse 차원에서 발생하는 거리 차이와, 각도-Doppler의 경 우, pulse 차원에서 발생하는 각도 차이로 인해 bias 오차 가 발생하게 되는데, 거리-각도의 경우와 본질적으로 같 은 문제이기 때문이다.

\section{FMCW MIMO 레이다 신호 모델}

단일 안테나에서, $n$ 번째 시간에서의 deramped된 FMCW 레이다 신호는 다음과 같이 주어진다.

$$
\begin{aligned}
& s[n]=a e^{j \phi} e^{j\left(2 \pi \gamma T_{s} n+2 \pi f_{c} \tau-\pi \gamma \tau^{2}\right)}, \\
& 0 \leq n \leq N-1,
\end{aligned}
$$

여기서 $a e^{j \phi}$ 는 표적의 반사율, $f_{c}$ 는 반송파 주파수, $\gamma$ 는 주 파수 증가 기울기, $T_{s}$ 는 샘플링 주기, $N$ 은 총 샘플링 수, $\tau$ 는 표적에서 수신안테나까지 걸리는 지연시간을 나타낸다.

송신배열 및 수신배열 소자 개수가 $P$ 개 및 $Q$ 개로 구성 된 $\mathrm{MIMO}$ 안테나는 $M=P Q$ 개의 가상수신배열(virtual receive array)로 나타낼 수 있다. 배열은 선형, 등간격이며, 인접 안테나 사이의 거리를 $d=\lambda / 2$, 배열의 중간인 origin 으로부터 표적까지의 거리를 $r$, 표적으로부터의 입사각 을 $\theta$ 라고 하자. 그러면, 표적으로부터 $m$ 번째 안테나까 지의 지연시간은 다음과 같다.

$$
\tau[m]=(2 r+d[m] u) / c,
$$

여기서, $d[m]=(m-(M-1) / 2) d$ 이며, 배열의 origin을 기준으로 $m$ 번째 안테나의 위치를 나타내고, $c$ 는 빛의 속 도이며, $u=\sin \theta$ 이다. 즉, $m$ 번째 안테나에서 deramped 된 $\mathrm{FMCW}$ 레이다 신호는 다음과 같다.

$$
\begin{aligned}
& s[n, m]=a e^{j\left(\phi-\pi \gamma \tau^{2}[m]\right)} e^{j\left(2 \pi f_{c} \tau[m]+2 \pi \gamma \tau[m] T_{s} n\right)} \\
& =a e^{j\left(\phi-\pi \gamma \tau^{2}[m]+4 \pi f_{c} r / c\right)} e^{j 2 \pi f_{c} d[m] u / c} e^{j 2 \pi \gamma(2 r+d[m] u) / c T_{s} n} \\
& \approx a e^{j \psi} e^{j 2 \pi \frac{u}{\lambda} d[m]} e^{j 2 \pi(2 r+d[m] u) \frac{B}{c N} n}
\end{aligned}
$$

여기서, 식 (5)에서 주파수 기울기 $\gamma$ 과 신호대역폭 $B$ 사 이의 관계인 $\gamma=B /\left(N T_{s}\right)$ 를 이용하였고, $\pi \gamma \tau^{2}[m]$ 은 $m$ 에 거의 의존적이지 않으므로, $\psi \approx \phi-\pi \gamma \tau^{2}[m]+$ $4 \pi f_{c} r / c$ 로 근사하였다.

$n$ 번째 시간, $m$ 번째 안테나에서 얻어지는 측정값은 다 음과 같다.

$$
z[n, m]=s[n, m]+w[n, m],
$$

여기서, $w[n, m] \sim C N\left(0, \sigma^{2}\right)$ 은 복소수 부가가우시안 잡음(additive Gaussian noise)으로 모델링하였다.

식 (3)에서 두 번째와 세 번째의 exponential term은 각 각 각도와 거리를 나타낸다. 하지만, 세 번째 exponential term에 각도의 요소인 $d[m] u$ 가 포함되어 있으며, 거리와 각도가 서로 결합(coupled)되어 있는 것을 알 수 있다. 따 라서 위 신호 모델에 2차원 주파수 추정기법을 적용하게 되면 bias 오차가 발생하게 된다.

\section{III. 제안하는 추정 알고리즘}

최대우도추정(maximum likelihood estimation: MLE) 기 법을 통해 $r$ 과 $u$ 를 추정함과 동시에, nuisance 변수인 $\psi$ 와 $a$ 도 함께 추정하는 것이 목표이다. 신호모델을 다시 써보면 다음과 같다.

$$
s[n, m]=a e^{j \psi} e^{j 2 \pi(u / \lambda) d[m]} g[n, m],
$$

여기서, $g[n, m]=e^{j 2 \pi(2 r+d[m] u) \frac{B}{c N} n}$ 이다. 식 (6) 측정 값을 시간 $(n)$ 축으로 쌓아서 열벡터 $z^{(m)} \in \mathbb{C}^{N \times 1}$ 를 만 들면 다음과 같다.

$$
\boldsymbol{z}^{(m)}=\boldsymbol{s}^{(m)}+\boldsymbol{w}^{(m)},
$$

여기서, 각 벡터값은 다음과 같다. 


$$
\begin{aligned}
& \boldsymbol{s}^{(m)}=a e^{j \psi} e^{j 2 \pi(u / \lambda) d[m]} g^{(m)}, \\
& \boldsymbol{g}^{(m)}=[g[0, m], g[1, m], \ldots, g[N-1, m]]^{T}, \\
& \boldsymbol{w}^{(m)}=[w[0, m], w[1, m], \ldots, w[N-1, m]]^{T} .
\end{aligned}
$$

그러면, 우도함수는 다음과 같이 주어진다.

$$
\Lambda_{0}=\sum_{m=0}^{M-1}\left[z^{(m)}-s^{(m)}\right]^{H} \cdot \frac{1}{\sigma^{2}} I \cdot\left[z^{(m)}-s^{(m)}\right]
$$

여기서, 윗첨자 $H$ 는 켤레전치(conjugate transpose)를 나타 낸다. 식 (7)을 식 (10)에 넣어 불필요한 부분을 제거하면 다음과 같아진다.

$$
\begin{aligned}
& \Lambda(a, \psi, r, u)= \\
& \quad-2 a \sum_{m=0}^{M-1} \operatorname{Re}\left[e^{j\left(\psi+2 \pi \frac{u}{\lambda} d[m]\right)} R^{(m)}\right]+M N a^{2}
\end{aligned}
$$

여기서, $\boldsymbol{R}^{(m)}$ 은 다음과 같이 주어진다.

$$
\begin{aligned}
R^{(m)}=z^{(m)^{H}} \cdot g^{(m)} & = \\
& \sum_{n=0}^{N-1} z^{*}[n, m] e^{j 2 \pi(2 r+d[m] u) \frac{B}{c N} n}
\end{aligned}
$$

우도함수 $\Lambda(\cdot)$ 를 최대로 만드는 변수 $\psi, a, u, r$ 을 찾 는 것이 목표이다.

첫 번째로, $\frac{\partial \Lambda}{\partial \psi}=0$ 을 만족하는 $\hat{\psi}$ 를 구해 보자.

$\frac{\partial \Lambda}{\partial \psi}=2 a \sum_{m=0}^{M-1} \operatorname{Im}\left[e^{j \psi} e^{j 2 \pi \frac{u}{\lambda} d[m]} R^{(m)}\right]$.

즉, $\hat{\psi}=-\measuredangle\left[\sum_{m=0}^{M-1} e^{j 2 \pi \frac{u}{\lambda} d[m]} R^{(m)}\right]$ 이다.

두 번째로, $\frac{\partial \Lambda}{\partial a}=0$ 을 만족하는 $\hat{a}$ 를 구해 보자.

$\frac{\partial \Lambda}{\partial a}=-2 \sum_{m=0}^{M-1} \operatorname{Re}\left[e^{j\left(\psi+2 \pi \frac{u}{\lambda} d[m]\right)} R^{(m)}\right]+2 M N a$.

즉, $\hat{a}=\frac{1}{M N} \sum_{m=0}^{M-1} \operatorname{Re}\left[e^{j\left(\psi+2 \pi \frac{u}{\lambda} d[m]\right)} R^{(m)}\right]$ 이다.

세 번째로, Newton-Raphson 기법을 이용하여 $\hat{u}$ 의 해를 찾는다.

$$
\hat{u}(i+1)=\hat{u}(i)-\left.\left(f_{u} / f_{u}^{\prime}\right)\right|_{\hat{u}=\hat{u}(i)},
$$

여기서, $\hat{u}(i)$ 는 $i$ 번째 반복에서의 $\hat{u}$ 를 나타내고, $f_{u}$ 와 $f_{u}^{\prime}$ 는 $u$ 에 대한 $\Lambda(\cdot)$ 의 Jacobian과 Hessian이며, 다음과 같이 구해진다.

$$
\begin{aligned}
& f_{u}=\frac{4 \pi}{\lambda} a \sum_{m=0}^{M-1} m \operatorname{Im}\left[e^{j\left(\psi+2 \pi \frac{u}{\lambda} d[m]\right)} R^{(m)}\right] \\
& -2 a \sum_{m=0}^{M-1} \operatorname{Re}\left[e^{j\left(\psi+2 \pi \frac{u}{\lambda} d[m]\right)} \frac{\partial R^{(m)}}{\partial u}\right] \\
& f_{u}^{\prime}= \\
& 2\left(\frac{2 \pi}{\lambda}\right)^{2} a \sum_{m=0}^{M-1} d^{2}[m] \operatorname{Re}\left[e^{j\left(\psi+2 \pi \frac{u}{\lambda} d[m]\right)} R^{(m)}\right] \\
& +4\left(\frac{2 \pi}{\lambda}\right) a \sum_{m=0}^{M-1} d[m] \operatorname{Im}\left[e^{j\left(\psi+2 \pi \frac{u}{\lambda} d[m]\right)} \frac{\partial R^{(m)}}{\partial u}\right. \\
& -2 a \sum_{m=0}^{M-1} \operatorname{Re}\left[e^{j\left(\psi+2 \pi \frac{u}{\lambda} d[m]\right)} \frac{\partial^{2} R^{(m)}}{\partial u^{2}}\right] .
\end{aligned}
$$

네 번째로, $\hat{r}$ 도 Newton Raphson 기법을 이용하여 구한다.

$$
\hat{r}(i+1)=\hat{r}(i)-\left.\left(f_{r} / f_{r}^{\prime}\right)\right|_{\hat{r}=\hat{r}(i)},
$$

여기서, $\hat{r}(i)$ 는 $i$ 번째 반복에서의 $\hat{r}$ 를 나타내고, $f_{r}$ 와 $f_{r}^{\prime}$ 는 $r$ 에 대한 $\Lambda(\cdot)$ 의 Jacobian과 Hessian이다.

$$
\begin{aligned}
& f_{r}=-2 a \sum_{m=0}^{M-1} \operatorname{Re}\left[e^{j\left(\psi+2 \pi \frac{u}{\lambda} d[m]\right)} \frac{\partial R^{(m)}}{\partial u}\right], \\
& f_{r}^{\prime}=-2 a \sum_{m=0}^{M-1} \operatorname{Re}\left[e^{j\left(\psi+2 \pi \frac{u}{\lambda} d[m]\right)} \frac{\partial^{2} R^{(m)}}{\partial u^{2}}\right] .
\end{aligned}
$$

여가서 $\partial R^{(m)} / \partial u, \partial^{2} R^{(m)} / \partial u^{2}, \partial R^{(m)} / \partial r, \partial^{2} R^{(m)} /$ $\partial r^{2}$ 는 식 (11)로부터 쉽게 구할 수 있다.

\section{IV. 모의실험 결과}

설정된 변수값은 반송파 주파수 $f_{c}=77 \mathrm{GHz}$, 신호대역 폭 $B=4 \mathrm{GHz}$, sweep time $S=10^{-4} \mathrm{~s}$ 이며, 거리 분해능은 $\Delta r=c /(2 B)=3.75 \mathrm{~cm}$ 이다. 거리 sample 개수 $N=256$, 송 신 안테나 개수 $P=4$, 수신 안테나 개수 $Q=4$ 이며, 가상 
배열 안테나 개수는 $M=P Q=16$ 이다. 표적은 거리 $r=5$ $\mathrm{m}$, 각도 $\theta=15^{\circ}$ 에 위치해 있다고 가정하였다.

2D-FFT에서 거리축과 각도축으로 $\times N_{z}(=2,048), \times M_{z}$ $(=2,048)$ 만큼 zero-padding을 하고, 2D-MUSIC에서는 거리 축과 각도축으로 $\times N_{z}(=2,048), \times M_{z}(=2,048)$ 만큼 미세 탐색(fine search)를 진행하였다. 이와 같이 oversampling을 한 이유는 기존의 알고리즘인 2D-FFT나 2D-MUSIC는 격 자기반(grid-based)이므로, 제안하는 무격자기반(gridlessbased) 알고리즘과 공평하게 비교하기 위해서이다. 따라 서 얻어지는 거리와 각도의 겉보기 분해능(apparent resolution)은 각각 $\Delta r / N_{z}=1.83 \times 10^{-5} \mathrm{~m}$ 와 $\Delta u /(d u / d \theta) / M_{z}=$ $2 /\left(M \cos \left(15^{\circ}\right)\right) \times 180 / \pi / M_{z}=0.0036^{\circ}$ 이다. 여기서 표적이 $\theta=$ $15^{\circ}$ 에 위치해 있기때문에 해당 각도에서 분해능을 구한 것이다. 만약, 격자기반 알고리즘의 평균제곱근편차(root mean square error: RMSE)가 겉보기 분해능(apparent resolution)값인 $1.83 \times 10^{-5} \mathrm{~m}$ 와 $0.0036^{\circ}$ 보다 크다면, 그 오차는 분해능의 부족 때문이 아니라, bias 오차 때문이다.

그림 1은 신호 대 잡음비(signal to noise ratio: SNR)에 따라 거리와 각도 추정치의 RMSE를 나타낸 것이다. 이 때, $\mathrm{SNR}=\mathrm{Pa}^{2} / \sigma^{2}$ 으로 정의하였다. 모의실험은 300 번 진 행한 평균 결과 값이고, $\psi \sim U(0,2 \pi)$ 가 균일분포를 갖 는다고 가정하였다. $2 \mathrm{D}-\mathrm{MUSIC}$ 에서 $L_{1} \times L_{2}(=10 \times 10)$ 부 행렬(submatrix)을 이용하여 spatial smoothing 기법 [2]을 사 용하였다. 2D-FFT 기법에서 거리의 bias 오차는 없으며, 각도의 bias 오차는 $0.397^{\circ}$ 이고, 2D-MUSIC 기법에서 거리 와 각도의 bias 오차는 $7.8 \times 10^{-4} \mathrm{~m}, 0.397^{\circ}$ 이다. 발생하는 오차값은 겉보기 분해능값인 $1.83 \times 10^{-5} \mathrm{~m}$ 와 $0.0036^{\circ}$ 보다 훨씬 크며, 이 오차는 분해능 부족 때문이 아닌, bias 오차 임을 알 수 있다. 표적이 $r=5 \mathrm{~m}, \theta=15^{\circ}$ 에 위치해 있을 경 우, Euclidean 거리오차는 $3.5 \mathrm{~cm}$ 로, 각도오차로 인한 위치 추정 오차가 큰 것을 알 수 있다. 반면, 제안하는 알고리 즘의 RMSE는 Cramer-Rao Bound와 일치함을 알 수 있다.

$N_{z}, M_{z}$ 만큼 zero-padding한 2D-FFT의 계산량은 $O$ $\left(M N M_{z} N_{z} \log _{2}\left(M N M_{z} N_{z}\right)\right)=5.84 \times 10^{11}$ 이며, $L\left(=L_{1} L_{2}=\right.$ 100) by $L$ 행렬의 eigenvalue decomposition과 $M_{z}$ by $N_{z}$ 만 큼 미세탐색으로 이루어진 2D-MUSIC 기법의 계산량은 $O\left(L^{3}+\right.$ $\left.M_{z} N_{z}\left(2 L^{2}+3 L-1\right)\right)=8.51 \times 10^{10}$ 이다. 제안하는 알고리즘 은 식 (12), (13), (14), (17)을 총 $I(=30)$ 번 반복하였으며, $O(I$

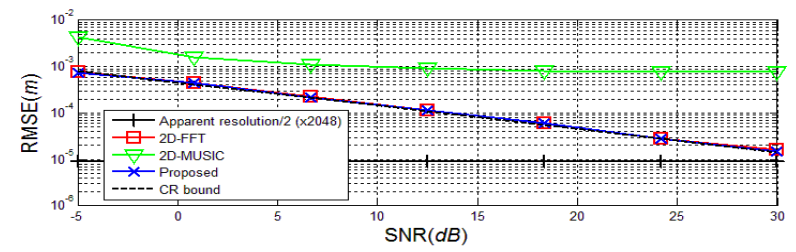

(a) 거리 추정치

(a) The range estimates

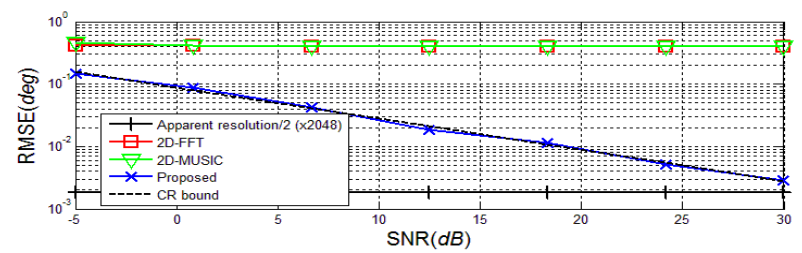

(b) 각도 추정치

(b) The angle estimates

그림 1. 표적의 평균 제곱근 편차(RMSE)

Fig. 1. RMSEs for the target.

$(46 M N+67 M+7))=5.68 \times 10^{6}$ 의 계산량으로 가장 작았다.

\section{$\mathrm{V}$. 결 론}

$\mathrm{FMCW}$ 배열을 이용하여 표적의 거리-각도를 추정할 경우, 기존의 2D-FFT 알고리즘을 적용하면 bias 오차가 나는 것을 모의실험을 통해 확인하였다. 이러한 bias 오차 를 극복하기 위해 최대우도법 알고리즘을 제안하였고, 제 안된 알고리즘은 $\mathrm{CRB}$ 에 도달함을 검증하였다. 레이다 기 반 SLAM 문제에서 bias 오차가 누적되어 차량의 위치가 크게 어긋나게 되는데, 본 알고리즘은 이와 같이 고정밀 추정이 요구되는 응용분야에 적용될 수 있다.

\section{References}

[1] 이혁중, 전주환, 송성찬, "밀리미터파(W밴드) FMCW $\mathrm{SAR}$ 기반 전방의 이동지상표적 탐지 및 위치와 속도 추정," 한국전자파학회논문지, 28(6), pp. 459-469, 2017년 6월.

[2] F. Belfiori, W. van Rossum, and P. Hoogeboom, "2DMUSIC technique applied to a coherent FMCW MIMO radar," in IET International Radar Conference on Radar Systems(Radar 2012), Glasgow, UK, pp. 1-6, Oct. 2012. 